\title{
Efeito do Lactobacillus reuteri na cólica infantil: revisão baseada na evidência
}

Carla Ribeiro, ${ }^{1}$ Sofia Coutinho ${ }^{2}$

\section{RESUMO}

Objetivo: Rever a evidência existente acerca da eficácia da suplementação com Lactobacillus reuteri na redução da sintomatologia das cólicas infantis.

Fontes de dados: National Guideline Clearinghouse, Guideline Finder, Canadian Medical Association, The Cochrane Database, DARE, Bandolier e MEDLINE/PubMed.

Métodos de revisão: Pesquisa de normas de orientação clínica, meta-análises, revisões sistemáticas e ensaios clínicos aleatorizados e controlados, entre agosto de 2005 e agosto de 2015, nas línguas portuguesa e inglesa. Foi utilizada a seguinte combinação de termos MeSH: 'Lactobacillus reuteri' e 'Colic'. Para atribuição dos níveis de evidência e forças de recomendação utilizou-se a escala Strength of Recommendation Taxonomy.

Resultados: Foram encontrados cinquenta e nove artigos, dos quais oito cumpriam os critérios de inclusão: duas revisões sistemáticas e seis ensaios clínicos aleatorizados e controlados. Cinco dos estudos incluídos e as duas revisões sistemáticas apresentaram resultados estatisticamente significativos na melhoria das cólicas. Apenas um estudo não demonstrou eficácia dos Lactobacillus reuteri na cólica infantil.

Conclusões: Após a análise dos artigos incluídos concluiu-se que existe evidência da suplementação com Lactobacillus reuteri na melhoria dos sintomas das cólicas infantis, comparativamente com o placebo (Força de Recomendação A). Considera-se que são necessários mais estudos nesta área, com boa qualidade, que validem a evidência encontrada e auxiliem na formulação de recomendações.

Palavras-chave: Lactobacillus reuteri; Cólica infantil.

\section{INTRODUÇÃO}

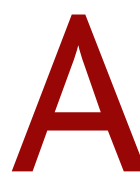
cólica infantil é uma condição benigna e autolimitada, que constitui um problema para os pais e cuidadores, associando-se a elevados níveis de stress e ansiedade parental, com impacto significativo nas crianças e suas famílias. ${ }^{1-2}$

Inicialmente, a cólica infantil foi definida como choro paroxístico e incontrolável num lactente saudável e bem alimentado com idade inferior a três meses, com duração superior a três horas por dia, em mais de três dias por semana e durante mais de três semanas. ${ }^{1-6} \mathrm{Re}-$ centemente, segundo os critérios Roma III para pato-

1Médica Interna de Medicina Geral e Familiar. USF La Salette, Centro de Saúde de Oliveira de Azeméis, ACES Entre Douro e Vouga II.

${ }^{2}$ Médica Interna de Medicina Geral e Familiar. UCSP Sul, Centro de Saúde de Oliveira de Azeméis, ACES Entre Douro e Vouga II. logias gastrointestinais funcionais, as cólicas infantis definem-se como paroxismos de irritabilidade, agitação ou choro, que começam e cessam sem causa óbvia, com duração igual ou superior a três horas por dia, ocorrendo pelo menos três dias por semana, durante pelo menos uma semana, sem que exista atraso no desenvolvimento. ${ }^{4,7-8}$

Estima-se que ocorra em cerca de $20 \%$ dos lactentes. ${ }^{2-4,9} \mathrm{~A}$ incidência é igual em ambos os sexos e não difere entre lactentes amamentados com leite materno ou com leite adaptado. ${ }^{1-2,4,8}$ As cólicas parecem estar associadas a baixo peso à nascença, primeiro filho, idade materna e tabagismo. ${ }^{1-2,6}$

As cólicas infantis são mais comuns nas primeiras semanas de vida, sendo mais intensas entre as quatro e as seis semanas, melhorando gradualmente até aos quatro/cinco meses. ${ }^{2,5,9}$ Durante cada episódio, o lac- 
tente parece incomodado, irritável e agitado, mas o sintoma mais marcante é o choro intenso, persistente e excessivo, com um tom mais elevado que o habitual e que tende a ocorrer principalmente ao anoitecer. ${ }^{3-5,8-9} \mathrm{O}$ lactente não responde às tentativas de consolo, nem mesmo pela amamentação. Pode exibir outros sintomas, como flexão das pernas contra o abdómen, rubor facial, punhos fechados e flatulência. ${ }^{2-5,9}$

A etiologia das cólicas infantis permanece desconhecida. ${ }^{1,-4,8,10}$ No entanto, têm sido propostas múltiplas hipóteses, incluindo alterações funcionais gastrointestinais, como hiperperistaltismo, desequilíbrio da microflora gastrointestinal, intolerância alimentar ou alergia às proteínas do leite. ${ }^{1-4,6,8,10}$

Da mesma forma, uma série de terapias têm sido tentadas, incluindo o uso de fórmulas hidrolisadas, de soja, com redução de lactose ou enriquecidas em fibras, sacarose, chás de ervas, musicoterapia, vibração ou massagem e manipulação da coluna vertebral; no entanto, nenhuma destas terapias provou ser efetiva. Tem sido sugerido que a cólica infantil aumenta a suscetibilidade à dor abdominal recorrente, doenças alérgicas e distúrbios psicológicos na infância. Evidências recentes sugerem que os probióticos podem oferecer algum benefício. ${ }^{11-12}$

Assim, o objetivo desta revisão é rever a evidência existente acerca da eficácia da suplementação com $L a c$ tobacillus reuteri (L reuteri) na redução da sintomatologia das cólicas infantis.

\section{MÉTODO}

Pesquisa bibliográfica em bases de dados sugeridas em artigos nacionais e internacionais sobre elaboração de revisões baseadas na evidência, nomeadamente: National Guidelines Clearinghouse, Guidelines Finder, Canadian Medical Association, The Cochrane Database, DARE, Bandolier e MEDLINE/PubMed.

Os descritores Medical Subject Headings (MeSH) utilizados foram: 'Lactoba- cillus reuteri' e 'Colic'. Incluíram-se normas de orientação clínica, revisões sistemáticas, meta-análises e ensaios clínicos aleatorizados, publicados entre agosto de 2005 e agosto de 2015, escritos em português ou inglês. Os artigos selecionados para leitura integral foram lidos pelas duas autoras e a atribuição dos níveis de evidência foi discutida unanimemente.

Os critérios de inclusão utilizados nesta revisão foram: 1) população: lactentes com cólicas infantis; 2) intervenção: utilização de $L$ reuteri; 3) comparação: placebo; 4) outcome: efeito dos probióticos nas cólicas infantis.

Definiram-se como critérios de exclusão: 1) evidência clínica de doença crónica ou patologia gastrointestinal; 2) ingestão de outros probióticos; e 3) ingestão de antibióticos na semana anterior.

Para a classificação dos níveis de evidência (NE) e a atribuição da força de recomendação (FR) utilizou-se a escala Strength of Recommendation Taxonomy (SORT), da American Academy of Family Physicians.

\section{RESULTADOS}

Na pesquisa bibliográfica inicial obtiveram-se cinquenta e nove artigos, sendo que oito artigos foram selecionados para a revisão (duas revisões sistemáticas e seis ensaios clínicos aleatorizados e controlados). O organigrama de seleção dos artigos está representado na figura 1. Os resultados dos mesmos estão sumariados nos quadros I e II.

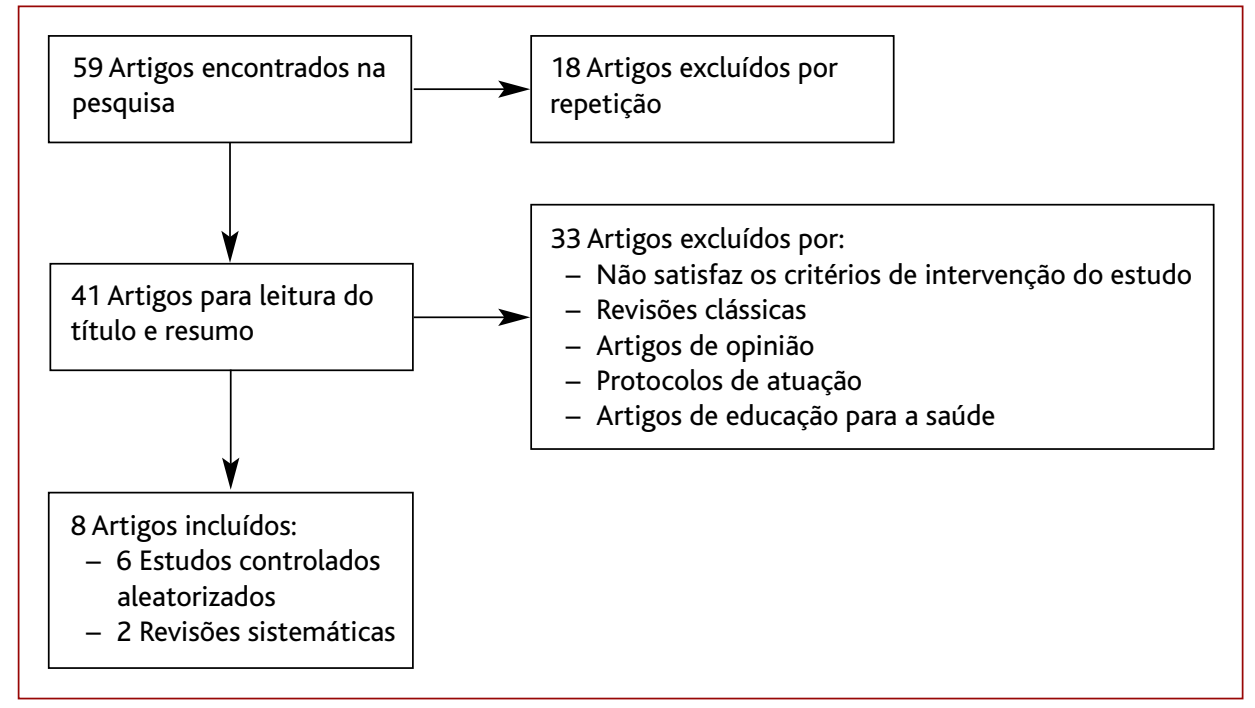

Figura 1. Organigrama de seleção dos artigos. 


\begin{tabular}{|c|c|c|c|c|c|c|}
\hline Referência & $\begin{array}{l}\text { Tipo de } \\
\text { estudo }\end{array}$ & População & $n$ & Intervenção & Resultados & $\begin{array}{l}\text { Nível de } \\
\text { Evidência }\end{array}$ \\
\hline $\begin{array}{l}\text { Savino F, } \\
\text { et al } 2010^{12}\end{array}$ & $\begin{array}{l}\text { ECA, } \\
\text { duplo-cego }\end{array}$ & $\begin{array}{l}\text { Lactentes com } \\
\text { cólica infantil, } \\
\text { exclusivamente } \\
\text { amamentados }\end{array}$ & $\begin{array}{l}46 \\
\text { lactentes }\end{array}$ & $\begin{array}{l}\text { L reuteri DSM } \\
17938,5 \text { gotas } \\
\text { orais, } 1 \text { vez/dia } \\
(n=25) \text { vs placebo } \\
(n=21) \text { durante } \\
21 \text { dias }\end{array}$ & $\begin{array}{l}\text { No grupo de tratamento, o } \\
\text { uso de } L \text { reuteri DSM } 17938 \\
\text { nas crianças exclusivamente } \\
\text { amamentadas resultou na } \\
\text { redução da sintomatologia } \\
\text { das cólicas infantis ( } 24 \text { vs } \\
15, p=0,036 \text { ) }\end{array}$ & 1 \\
\hline $\begin{array}{l}\text { Szajewska H, } \\
\text { et al } 2013^{11}\end{array}$ & $\begin{array}{l}\text { ECA, } \\
\text { duplo-cego }\end{array}$ & $\begin{array}{l}\text { Lactentes com }<5 \\
\text { meses com cólica } \\
\text { infantil, exclusiva ou } \\
\text { predominantemente } \\
\text { amamentados }\end{array}$ & $\begin{array}{l}80 \\
\text { lactentes }\end{array}$ & $\begin{array}{l}\text { L reuteri DSM } \\
17938,5 \text { gotas } \\
\text { orais, } 1 \text { vez/dia } \\
(n=40) \text { vs placebo } \\
(n=40) \text { durante } \\
21 \text { dias }\end{array}$ & $\begin{array}{l}\text { No grupo de tratamento, o } \\
\text { uso de } L \text { reuteri DSM } 17938 \\
\text { resultou na redução da } \\
\text { sintomatologia das cólicas } \\
\text { infantis ( } R R 2,7,95 \% \text { Cl } \\
1,85-4,1, P<0,001 \text { ) }\end{array}$ & 1 \\
\hline $\begin{array}{l}\text { Sung V, et al } \\
2014^{13}\end{array}$ & $\begin{array}{l}\text { ECA, } \\
\text { duplo-cego }\end{array}$ & $\begin{array}{l}\text { Lactentes nos } 3 \\
\text { primeiros meses }\end{array}$ & $\begin{array}{l}167 \\
\text { lactentes }\end{array}$ & $\begin{array}{l}\text { L reuteri DSM } \\
17938,5 \text { gotas } \\
\text { orais, } 1 \text { vez/dia } \\
(n=85) \text { vs placebo } \\
(n=82) \text { durante } 6 \\
\text { meses }\end{array}$ & $\begin{array}{l}\text { Os lactentes amamentados } \\
\text { não beneficiaram do uso de } \\
\text { L reuteri DSM } 17938 \\
(p=0,02)\end{array}$ & 2 \\
\hline $\begin{array}{l}\text { Indrio } F \text {, et al } \\
2014^{14}\end{array}$ & $\begin{array}{l}\text { ECA, } \\
\text { duplo-cego }\end{array}$ & $\begin{array}{l}\text { Lactentes nos } 3 \\
\text { primeiros meses }\end{array}$ & $\begin{array}{l}468 \\
\text { lactentes }\end{array}$ & $\begin{array}{l}\text { L reuteri DSM } \\
17938,5 \text { gotas } \\
\text { orais, } 1 \text { vez/dia } \\
(n=238) \text { vs placebo } \\
(n=230) \text { durante } \\
90 \text { dias }\end{array}$ & $\begin{array}{l}\text { O uso de } L \text { reuteri DSM } \\
17938 \text { reduziu a } \\
\text { sintomatologia de cólicas } \\
\text { infantis }(37,7 \mathrm{~min} / \mathrm{d} \text { vs } 70,9 \\
\text { min/d; } P<0,01) \text {, o tempo de } \\
\text { choro ( } 38 \text { vs } 71 \text { min; } p<0,01) \text {, } \\
\text { o número médio de } \\
\text { regurgitações ( } 2,9 \text { vs } 4,9 ; \\
p<0,01) \text { e aumentou o } \\
\text { número de dejeções por dia } \\
(4,2 \text { vs } 3,6 ; p<0,01)\end{array}$ & 1 \\
\hline $\begin{array}{l}\text { Chau K, Lau } \\
\text { E et al } \\
2015^{15}\end{array}$ & $\begin{array}{l}\text { ECA, } \\
\text { duplo-cego }\end{array}$ & $\begin{array}{l}\text { Lactentes com } 3 \text { a } 6 \\
\text { meses }\end{array}$ & $\begin{array}{l}52 \\
\text { lactentes }\end{array}$ & $\begin{array}{l}\text { L reuteri DSM } \\
17938,5 \text { gotas } \\
\text { orais, } 1 \text { vez/dia } \\
(n=24) \text { vs placebo } \\
(n=28) \text { durante } \\
21 \text { dias }\end{array}$ & $\begin{array}{l}\text { O uso de } L \text { reuteri DSM } \\
17938 \text { nas crianças } \\
\text { amamentadas resultou na } \\
\text { redução da sintomatologia } \\
\text { das cólicas infantis (RR } 3,3 \text {, } \\
95 \% \mathrm{Cl} 1,55-7,03, p=0,035 \text { ) }\end{array}$ & 1 \\
\hline
\end{tabular}

Em 2010, Savino e colaboradores ${ }^{12}$ realizaram um estudo controlado aleatorizado, duplo-cego, com o objetivo de testar a eficácia de $L$ reuteri na cólica infantil e avaliar a sua relação com a microflora intestinal. A amostra incluiu 46 lactentes com cólica infantil, exclu- sivamente amamentados. Foi administrado L reuteri DSM 17938, cinco gotas orais uma vez por dia $(n=25) v s$ placebo ( $n=21)$ durante 21 dias. Observou-se que, no grupo tratado, o uso de $L$ reuteri DSM 17938, nas crianças exclusivamente amamentadas, resultou na redu- 


\begin{tabular}{|c|c|c|c|c|c|c|}
\hline Referência & $\begin{array}{l}\text { Tipo de } \\
\text { estudo }\end{array}$ & População & $n$ & Intervenção & Resultados & $\begin{array}{l}\text { Nível de } \\
\text { Evidência }\end{array}$ \\
\hline $\begin{array}{l}\text { Savino F, } \\
\text { et al } 2015^{16}\end{array}$ & ECA & $\begin{array}{l}\text { Lactentes com }<10 \\
\text { dias }\end{array}$ & $\begin{array}{l}105 \\
\text { lactentes }\end{array}$ & $\begin{array}{l}\text { L reuteri DSM } \\
17938,5 \text { gotas } \\
\text { orais, } 1 \text { vez/dia } \\
(n=51) \text { vs placebo } \\
(n=53) \text { durante } \\
12 \text { semanas }\end{array}$ & $\begin{array}{l}\text { O uso de } L \text { reuteri DSM } \\
17938 \text { está associado à } \\
\text { redução do número de } \\
\text { consultas pediátricas devido } \\
\text { a cólica infantil ( } p<0,0001 \text { ), } \\
\text { bem como da utilização dos } \\
\text { fármacos para alívio da dor } \\
\text { (simeticone RR } 0,2495 \% \mathrm{Cl} \\
0,14-0,41, p<0,0001 \text { e NNT } \\
1,49 \text { ) e fórmula para lactentes } \\
\text { (RR } 0,3795 \% \text { Cl } 0,17-0,80 \text {, } \\
p=0,0121 \text { NNT } 4,29 \text { ) }\end{array}$ & 2 \\
\hline
\end{tabular}

Legenda: $\mathrm{Cl}$ - intervalo de confiança; ECA - Estudo controlado e aleatorizado; L. reuteri - Lactobacillus reuteri; NNT - number needed to treat; $\mathrm{RR}$ - risco relativo.

\section{QUADRO II. Revisões sistemáticas}

\begin{tabular}{|c|c|c|c|c|}
\hline $\begin{array}{l}\text { Anabrees J, } \\
\text { et al } 2013^{17}\end{array}$ & RS & $\begin{array}{l}3 \text { MT } \\
n=220 \text { lactentes amamentados exclusiva ou } \\
\text { predominantemente amamentados } \\
\text { L reuteri DSM } 17938,5 \text { gotas orais, } 1 \text { vez/dia } \\
\text { vs placebo ou vs simeticone durante } 21 \text { dias }\end{array}$ & $\begin{array}{l}\text { L reuteri DSM } 17938 \text { comparado com o placebo } \\
\text { aumenta significativamente o sucesso do } \\
\text { tratamento das cólicas infantis (RR 0,06 95\% } \\
\text { Cl 0,01-0,25 e NNT 2) }\end{array}$ & 1 \\
\hline $\begin{array}{l}\text { Urbanska M, } \\
\text { et al } 2014^{18}\end{array}$ & RS & $\begin{array}{l}3 \text { ECA } \\
n=257 \text { lactentes } \\
L \text { reuteri DSM } 17938,5 \text { gotas orais, } 1 \text { vez/dia } \\
\text { vs placebo durante } 21 \text { dias }\end{array}$ & $\begin{array}{l}\text { L reuteri DSM } 17938 \text { reduz o tempo de choro } \\
\text { (-43 min/d } 95 \% \mathrm{Cl}-68 \text { a }-19) \text {, principalmente } \\
\text { nos lactentes exclusiva ou predominantemente } \\
\text { amamentados (-57 } \mathrm{min} / \mathrm{d} 95 \% \mathrm{Cl}-67 \text { a }-46)\end{array}$ & 2 \\
\hline
\end{tabular}

Legenda: $\mathrm{Cl}$ - intervalo de confiança; ECA - Estudo controlado e aleatorizado; L. reuteri - Lactobacillus reuteri; MT - metanálise; NNT - number needed to treat; RR - risco relativo; RS - revisão sistemática.

ção da sintomatologia das cólicas infantis $(24$ vs 15 , $p=0,036)$. Trata-se de um estudo de elevada qualidade, pelo que se atribui um NE 1 .

Szajewska e colaboradores ${ }^{11}$ (2013) realizaram um estudo controlado aleatorizado, duplo-cego, com o objetivo de determinar se administração de $L$ reuteri DSM 17938 seria benéfica em lactentes com cólica infantil a ser amamentados. A amostra incluiu 80 lactentes com idade inferior a cinco meses, com cólica infantil, exclusiva ou predominantemente amamentados. Comparou-se a administração de $L$ reuteri DSM 17938, cin- co gotas orais uma vez por dia $(n=40) v$ s placebo $(n=40)$ durante 21 dias. Observou-se que o grupo tratado com $L$ reuteri DSM 17938 resultou na redução da sintomatologia das cólicas infantis (RR 2,7, 95\% CI 1,85-4,1, $p<0,001$ ), tendo-se atribuído um NE 1 , de acordo com os critérios SORT.

Com o objetivo de determinar se o probiótico $\mathrm{L} \mathrm{reu}$ teri DSM 17938 reduz o choro ou agitação, Sung e colaboradores ${ }^{13}$ realizaram um estudo controlado aleatorizado, duplo-cego. A amostra incluiu 167 lactentes amamentados e alimentados com fórmula, com idade in- 
ferior a três meses. Foi administrado L reuteri DSM 17938 , cinco gotas orais uma vez por dia $(n=85) v s$ placebo ( $n=82$ ) durante seis meses. Observou-se que os lactentes amamentados não beneficiaram do uso de $L$ reuteri DSM 17938 ( $p=0,02$ ). Foi atribuído um NE 2, devido ao facto de a população do estudo ser maioritariamente selecionada a partir de serviços de urgência, aspeto considerado pelos próprios autores como uma limitação.

Em 2014 foi realizado um estudo controlado aleatorizado, duplo-cego, por Indrio e colaboradores ${ }^{14}$ com o objetivo de investigar se a suplementação oral com $L$ reuteri DSM 17938 durante os primeiros três meses de vida pode reduzir o aparecimento de cólica, refluxo gastroesofágico, obstipação e, assim, reduzir o impacto socioeconómico dessas condições. A amostra incluiu 468 lactentes nos três primeiros meses de vida, aos quais foi administrado L reuteri DSM 17938, cinco gotas orais, uma vez por dia $(n=238)$ vs placebo $(n=230)$ durante 90 dias. Observou-se que no grupo em que foi usado o $L$ reuteri DSM 17938 houve redução da sintomatologia de cólicas infantis (37,7 min/dia $v s 70,9 \mathrm{~min} / \mathrm{dia}, p<0,01)$, do tempo de choro (38 min vs $71 \mathrm{~min}, p<0,01$ ), do número médio de regurgitações $(2,9 v s 4,9, p<0,01)$ e houve aumento do número de dejeções por dia $(4,2$ vs 3,6, $p<0,01)$. Foi atribuído um NE 1 .

Em 2015, Chau e colaboradores ${ }^{15}$ realizaram um estudo controlado aleatorizado, duplo-cego, com o objetivo de investigar a eficácia do L reuteri DSM 17938 no tratamento da cólica infantil em lactentes amamentados em comparação com o placebo. A amostra incluiu 52 lactentes entre os três e seis meses de idade. Foi administrado L reuteri DSM 17938, cinco gotas orais uma vez por dia $(n=24)$ vs placebo $(n=28)$ durante 21 dias. Observou-se que o uso de $L$ reuteri DSM 17938 nas crianças amamentadas resultou na redução da sintomatologia das cólicas infantis (RR 3,3, 95\% CI 1,55-7,03, $p=0,035)$, tendo sido atribuído um NE 1 por se tratar de um estudo de elevada qualidade.

Savino e colaboradores ${ }^{16}$ (2015) realizaram um estudo controlado aleatorizado, com o objetivo de avaliar se a administração de $L$ reuteri DSM 17938 com vitamina D3, antes dos 10 dias de vida, era eficaz para reduzir a cólica infantil e o desconforto dos pais de crianças saudáveis. A amostra incluiu 105 lactentes, com idade inferior a 10 dias. Foi administrado L reuteri DSM
17938, cinco gotas orais uma vez por dia no grupo de tratamento, que correspondia a 51 lactentes e placebo aos 53 lactentes do grupo de controlo durante 12 semanas. Observou-se que o uso de L reuteri DSM 17938 está associado à redução do número de consultas pediátricas devido a cólica infantil $(p<0,0001)$, bem como da utilização de fármacos para alívio da dor (simeticone RR 0,24, 95\% CI 0,14-0,41, $p<0,0001$ e NNT 1,49) e fórmula para lactentes (RR 0,37, 95\% CI 0,17-0,80, $p=0,0121$, NNT 4,29). Foi atribuído um NE 2 por não se ter feito uma dupla ocultação neste estudo.

Uma revisão sistemática, realizada por Anabrees e colaboradores, ${ }^{17}$ em 2013, incluiu três meta-análises, envolvendo no total 220 lactentes exclusiva ou predominantemente amamentados. Foi administrado o probiótico L reuteri DSM 17938, cinco gotas orais uma vez por dia $v s$ placebo $v s$ simeticone durante 21 dias. Observou-se que o grupo que usou L reuteri DSM 17938, quando comparado com o placebo, o probiótico aumenta significativamente o sucesso do tratamento das cólicas infantis (RR 0,06, 95\% CI 0,01-0,25 e NNT 2). Esta revisão sistemática apresenta um NE1.

Outra revisão sistemática, realizada em 2014 por Urbanska e colaboradores, ${ }^{18}$ incluiu três estudos controlados aleatorizados, já descritos anteriormente, envolveu 293 lactentes, aos quais foi administrado $L$ reuteri DSM 17938, cinco gotas orais uma vez por dia, $v$ placebo durante 21 dias. Observou-se que o grupo submetido a L reuteri DSM 17938 reduziu o tempo de choro (-43 min/dia, 95\% CI -68 e -19), principalmente nos lactentes exclusiva ou predominantemente amamentados (-57 min/dia, 95\% CI -67 a -46). Foi atribuído um NE 2 a esta revisão sistemática, dado que inclui um estudo prospetivo, não duplo-cego, o que pode influenciar os resultados encontrados.

\section{CONCLUSÕES}

Através da evidência disponível conclui-se que a suplementação com L reuteri melhora os sintomas das cólicas infantis, quando comparada com o placebo, com uma força de recomendação A. Esta revisão compreende estudos, a maioria de boa qualidade, o que proporciona robustez às conclusões.

As cólicas infantis são uma fonte de grande preocupação para os pais, sendo importante o papel do médico no aconselhamento e apoio. Inicialmente deve ser 
avaliada a técnica de aleitamento, sendo ainda importante tranquilizar os cuidadores e fornecer conselhos gerais, enfatizando a natureza autolimitada da situação.

Apesar de ainda existir pouca evidência que suporte o seu uso, os probióticos poderão ser tentados, especialmente nos casos mais severos, em que os pais têm dificuldades em lidar com a situação após aconselhamento e tranquilização.

Todos os pais devem ser aconselhados e encorajados a tentar modificações comportamentais, como: estimulação auditiva, massagem, tranquilização do lactente (uso de chupeta ou colo) e alteração da postura do lactente após a alimentação para uma posição mais vertical.

Apesar da maioria dos estudos incluídos nesta revisão mostrarem resultados estatisticamente significativos no uso de probióticos para a redução da sintomatologia das cólicas infantis, considera-se que são necessários mais estudos nesta área, com boa qualidade, que validem a evidência encontrada e auxiliem na formulação de recomendações.

\section{REFERÊNCIAS BIBLIOGRÁFICAS}

1. Drug and Therapeutics Bulletin. Management of infantile colic. BMJ. 2013;347:f4102.

2. Pray WS. Nonprescription product therapeutics. 2 nd ed. Philadelphia: Lippincott Williams \& Wilkins; 2005. ISBN 9780781734981

3. Roberts DM, Ostapchuk M, O'Brien JG. Infantile colic. Am Fam Physician. 2004;70(4):735-40.

4. Vandenplas Y, Gutierrez-Castrellon P, Velasco-Benítez C, Palacios J, Jaen $\mathrm{D}$, Ribeiro $\mathrm{H}$, et al. Practical algorithms for managing common gastrointestinal symptoms in infants. Nutrition. 2013;29(1):184-94.

5. Cohen-Silver J, Ratnapalan S. Management of infantile colic: a review. Clin Pediatr. 2009;48(1):14-7.

6. Evanoo G. Infant crying: a clinical conundrum. J Pediatr Health Care. 2007;21(5):333-8.

7. Critch JN. Infantile colic: is there a role for dietary interventions? Paediatr Child Health. 2011;16(1):47-9.

8. Ortega Páez E, Barroso Espadero D. Cólico del lactante. Rev Pediatr Aten Primaria. Supl. 2013;(22):81-7. Spanish
9. Colic and crying: self-care. MedlinePlus [Internet]. 2015 [updated 2015 Jul 10; cited 2015 Aug 31]. Available from: https://medlineplus.gov/ ency/patientinstructions/000753.htm

10. Perry R, Hunt K, Ernst E. Nutritional supplements and other complementary medicines for infantile colic: a systematic review. Pediatrics. 2011;127(4):720-33.

11. Szajewska H, Gyrczuk E, Horvath A. Lactobacillus reuteri DSM 17938 for the management of infantile colic in breastfed infants: a randomized, double-blind, placebo-controlled trial. J Pediatr. 2013;162(2):25762.

12. Savino F, Cordisco L, Tarasco V, Palumeri E, Calabrese R, Oggero R, et al. Lactobacillus reuteri DSM 17938 in infantile colic: a randomized, double-blind, placebo-controlled trial. Pediatrics. 2010;126(3):e526-33.

13. SungV, Hiscock H, Tang ML, Mensah FK, Nation ML, Satzke C, et al. Treating infant colic with the probiotics Lactobacillus reuteri: double blind, placebo controlled randomized trial. BMJ. 2014;348:g2107.

14. Indrio F, Di Mauro A, Riezzo G, Civardi E, Intini C, Corvaglia L, et al. Prophylactic use of a probiotic in the prevention of colic, regurgitation, and functional constipation: a randomized clinical trial. JAMA Pediatr. 2014;168(3):228-33.

15. Chau K, Lau E, Greenberg S, Jacobson S, Yazdani-Brojeni P, Verma N, et al. Probiotics for infantile colic: a randomized, double-blind, placebocontrolled trial investigating Lactobacillus reuteri DSM 17938.J Pediatr. 2015;166(1):74-8.

16. Savino F, Ceratto S, Poggi E, Cartosio ME, Cordero di Montezemolo L, Giannattasio A. Preventive effects of oral probiotic on infantile colic: a prospective, randomised, blinded, controlled trial using Lactobacillus reuteri DSM 17938. Benef Microbes. 2015;6(3):245-51.

17. Anabrees J, Indrio F, Paes B, AlFaleh K. Probiotics for infantile colic: a systematic review. BMC Pediatr. 2013;13:186.

18. Urbanska M, Szajewska H. The efficacy of Lactobacillus reuteri DSM 17938 in infants and children: a review of the current evidence. Eur J Pediatr. 2014;173(10):1327-37.

\section{CONFLITO DE INTERESSES}

Os autores declaram não ter conflitos de interesses.

\section{ENDEREÇO PARA CORRESPONDÊNCIA}

Carla Sofia Moreira Ribeiro

Rua do Nora, 265 Canidelo, 4400-513 Vila Nova de Gaia

E-mail: carlasmribeiro@hotmail.com

Recebido em 09-03-2016

Aceite para publicação em 07-10-2016 


\section{ABSTRACT}

\section{THE EFFECT OF LACTOBACILLUS REUTERI ON INFANTILE COLIC: AN EVIDENCE-BASED REVIEW}

Objective: To review the evidence for the effectiveness of probiotic supplementation with Lactobacillus reuteri in reducing symptoms of infantile colic.

Data sources: National Guideline Clearinghouse, Guideline Finder, Canadian Medical Association, The Cochrane Database, DARE, Bandolier and MEDLINE/PubMed.

Methods: A review of clinical guidelines, meta-analyses, systematic reviews, and randomized controlled clinical trials, published between August 2005 and August 2015, in both the Portuguese and English languages was conducted. The following MeSH terms were used: 'Lactobacillus reuteri' and 'Colic'. For the attribution of levels of evidence and the strength of recommendations, the Strength of Recommendation Taxonomy scale was used.

Results: Fifty-nine papers were found and eight fulfilled the inclusion criteria. These included two systematic reviews and six randomized controlled clinical trials. Five of the included studies and both systematic reviews found statistically significant improvements in symptoms of colic with probiotic supplementation. One study did not demonstrate the effectiveness of Lactobacillus reuteri in infantile colic.

Conclusion: There is evidence for improvement of symptoms of infantile colic with Lactobacillus reuteri supplementation, compared to placebo (Strength of Recommendation A). More good quality studies are necessary to validate these findings and to aid in the formulation of clinical guidelines.

Keywords: Lactobacillus reuteri; Infantile colic. 